\title{
Sous-titrage publicitaire pour sourds et malentendants: enjeux, défis et perspectives d'avenir
}

\author{
Isabel Cómitre Narváez ${ }^{1}$ \\ Universidad de Málaga
}

\begin{abstract}
Subtitling for deaf people has undergone a strong evolution in the last years thanks to the legislation and to the lobbies which conduct campaigns in favour of its development. However, as far as TV advertising, both in France and in Spain, are concerned, there is a lack of compulsory legislation on subtitles, which is the reason why some campaigns are inaccessible to the deaf community. This work aims to show the fundamental need for the translator of subtitles for deaf and hard of hearing people to reproduce the communication strategy of the brand and to adapt advertising messages in a creative and inclusive way. The commercial for the product Kinder Bueno by the Ferrero group constitutes a representative example of it. The challenge for the professional subtitler is to find a strategic compromise between the language of the brand and the targeted deaf community on which depends the success or failure of the advertising campaign.
\end{abstract}

Keywords: audiovisual translation, subtitling for the deaf and hard of hearing, advertising campaigns

\section{Résumé}

Le sous-titrage pour malentendants a subi une forte évolution ces dernières années grâce à la législation et aux groupes de pression qui mènent des campagnes en faveur de son développement. Néanmoins, en ce qui concerne la publicité télévisée en France et en

\footnotetext{
Corresponding author - Universidad de Málaga, Departamento de Traducción e Interpretación, Campus de Teatinos, s/n, 29071 Málaga (España).

Email: comitre@uma.es
} 
Espagne, il n'y a pas d'obligation réglementaire concernant le sous-titrage et certaines campagnes sont inaccessibles pour la communauté malentendante. Le présent travail a pour objectif de démontrer la nécessité fondamentale pour le traducteur de sous-titres pour sourds et malentendants de reproduire la stratégie de communication de la marque et d'adapter les messages publicitaires de façon créative et inclusive. Le spot pour le produit Kinder Bueno du groupe Ferrero en constitue un exemple représentatif. Le défi pour le soustitreur professionnel consiste à trouver un compromis stratégique entre le langage de la marque et le public cible demalentendants dont dépendra le succès ou l'échec de la campagne publicitaire.

Mots-clés: traduction audiovisuelle, sous-titrage pour sourds et malentendants, campagnes publicitaires

\section{Introduction}

Dans un monde dominé par l'audiovisuel -Internet, télévision numérique, DVD, podcasting- il n'est que justice que l'accès à ces contenus multimédias soit à la portée de tous. En ce qui concerne les personnes sourdes et malentendantes, l'accès à l'information et à la culture est possible grâce au sous-titrage pour sourds et malentendants, dorénavant sous-titrage SM (Neves, 2008). C'est une réalité tangible en Grande-Bretagne où, depuis des années, cette pratique est très répandue parmi les annonceurs ${ }^{2}$ et s'étend peu à peu à l'ensemble des pays européens. En France, le Conseil Supérieur de l'Audiovisuel (CSA) et la commission de développement durable de l'Association des Agences-Conseil en Communication $(\mathrm{AACC})^{3}$ ont développé des actions pour procéder au sous-titrage des spots publicitaires depuis l'entrée en vigueur de la loi du 11 février 2005 qui exige l'adaptation des programmes audio-visuels aux personnes sourdes et malentendantes. Même si cette loi ne s'étend pas aux messages publicitaires, de nombreux annonceurs ont pris des dispositions afin de toucher ce public qui constitue un pourcentage de consommateurs potentiels non négligeable. Depuis 2011, en France, I'Oréall ${ }^{4}$, Ferrero, BMW, GMT, AMF, et de nombreux annonceurs sous-titrent tous leurs spots pour les personnes sourdes et malentendantes. En Espagne, la Ley General de la Comunicación Audiovisual (31-3-2010), régule le secteur audiovisuel et n'envisage pas le sous-titrage des films publicitaires. Or, le présent travail a pour but de démontrer la nécessité de sous-titrer les films

$<$ http://www.uda.fr/sinformer-actualites/actualites/partenaires-

prestataires/productions/article/enfin-le-sous-titrage-\%20des-spots-pour-les-malentendants/>

3 <http://soustitronsnospublicites.aacc.fr/>

4 <http://www.medias-soustitres.com/television/actualites/article/l-oreal-generalise-le-soustitrage $>$ 
publicitaires pour un public malentendant avec une démarche plus créative d'inclusion. $^{5}$

\section{Le sous-titrage pour sourds et malentendants}

\subsection{Contexte réglementaire}

Nous l'avons déjà indiqué, l'enjeu principal de l'accessibilité concerne une large population. Selon un rapport publié en mars 2015, I'Organisation Mondiale de la Santé (OMS) signale que ${ }^{6}$ :

Plus de $5 \%$ de la population mondiale, soit 360 millions de personnes, souffre d'une déficience auditive incapacitante (perte d'audition supérieure à 40 décibels $(\mathrm{dB})$ dans la meilleure oreille chez l'adulte et à $30 \mathrm{~dB}$ dans la meilleure oreille chez l'enfant) - soit 328 millions d'adultes et 32 millions d'enfants. La plupart vivent dans des pays à revenu faible ou intermédiaire. Environ un tiers des personnes de plus de 65 ans sont touchées par une perte d'audition incapacitante. La prévalence de ce trouble dans cette tranche d'âge est la plus élevée en Asie du Sud, en Asie-Pacifique et en Afrique subsaharienne. Dans l'Union Européenne, on estime que plus de 55 millions de personnes sont déficients auditifs ${ }^{7}$.

En France, même s'il n'y a pas actuellement de source statistique officielle qui publie des chiffres totalement fiables ni de définition unique des seuils, on estime qu'environ $8 \%$ de la population souffre de déficience auditive à des degrés divers de moyenne à sévère ou légère. De plus, de nombreuses personnes ne reconnaissent pas spontanément mal entendre. On a constaté les mêmes proportions dans les principaux pays européens. La déficience auditive ne concerne pas seulement les seniors: les statistiques démontrent que la population est atteinte de plus en plus tôt.

Depuis 2010, le Conseil Supérieur de l'Audiovisuel (CSA), par le biais de la réglementation (Loi du 11/02/2005), dispose que le sous-titrage SM est généralisé sur toutes les grandes chaînes françaises publiques et privées: $100 \%$ des programmes des chaînes sont accessibles aux personnes sourdes et malentendantes à l'exception des messages publicitaires. Ceci peut être considéré

<http://www.medias-soustitres.com/television/actualites/article/faut-il-sous-titrer-les-publicites>

$6 \quad$ Surdité et déficience auditive. Organisation Mondiale de la Santé (OMS). mars 2015. <http://www.who.int/mediacentre/factsheets/fs300/fr/>.

7 Forum écoute $<$ http://www.ecoute.ch/Perte_Auditive_Statistiques_Malentendants_Chiffres $>$. 
comme une rupture car la diffusion de films publicitaires fait partie intégrante d'un support médiatique comme la télévision et l'accessibilité totale serait souhaitable.

\subsection{Objectifs et normes}

Le sous-titrage SM a subi une forte évolution ces dernières années grâce au succès des groupes de pression qui mènent des campagnes en faveur de son développement au bénéfice du public visé. L'objectif principal du sous-titrage SM est d'assurer l'intelligibilité de l'ensemble du contenu audio d'une émission de télévision ce qui englobe non seulement la parole, mais également la sensation auditive globale. De cet objectif découle l'importance d'une bonne lisibilité des sous-titres à l'écran. À cet effet, la réalisation du sous-titrage SM doit obéir à des normes et à des conventions qui varient selon les pays et qui veillent à la bonne lisibilité et à la qualité du sous-titrage SM. Ces normes et conventions imposent des contraintes notamment sur le nombre de caractères, la durée d'affichage, les règles de couleur et de symbolisation, etc. En Grande-Bretagne, les normes et conventions officielles sont celles du «style» $\mathrm{BBC}^{8}$ et elles ont influencé toute l'Europe. En France, ces contraintes sont formalisées dans la Charte relative à la qualité du sous-titrage à destination des personnes sourdes ou malentendantes ${ }^{9}$, promue par le Conseil Supérieur de l'Audiovisuel (CSA) après concertation avec les publics concernés. En Espagne, c'est la norme UNE 153010 Subtitulado para personas sordas y personas con discapacidad auditiva qui réglemente le sous-titrage SM. Cette norme a d'ailleurs été sévèrement critiquée par Pereira \& Lorenzo (2005) et Pereira (2010) car elle n'est pas adaptée aux besoins du public de malentendants si diversifié. La qualité du soustitrage SM est donc primordiale (Neves, 2008), tout particulièrement dans le contexte publicitaire où cela peut supposer l'échec ou le succès de la campagne.

\subsection{Spécificités}

Dans le cadre de la traduction audiovisuelle, De Linde (1996) faisait déjà une distinction entre deux formes de sous-titrage: le sous-titrage interlinguistique et le sous-titrage intralinguistique. Díaz Cintas (2008, pp. 27) reprend cette distinction selon laquelle ce dernier désigne

8 <http://www.bbc.co.uk/guidelines/futuremedia/accessibility/subtitling_guides/online_sub_editor ial_guidelines_vs1_1.pdf>

9 <http://www.csa.fr/Juridical-area/Chartes/Charte-relative-a-la-qualite-du-sous-titrage-adestination-des-personnes-sourdes-ou-malentendantes-Decembre-2011> 
une pratique de traduction qui consiste à présenter, en général sur la partie inférieure de l'écran, mais pas toujours, [...] un texte écrit qui s'attache à restituer:

1. le dialogue original des locuteurs, qu'ils soient ou non à l'écran

2. les éléments discursifs qui apparaissent à l'image (les lettres, les insertions, les grafitti, les pancartes, les écrans d'ordinateurs et tout ce qui est du même ordre)

3. d'autres éléments discursifs qui font partie de la bande son, comme les chansons, les voix émanant de postes de télévision, de radios ou d'ordinateurs, etc.»

Le sous-titrage intralinguistique qui «implique un passage de l'oral à l'écrit tout en restant au sein d'une même langue» (Díaz Cintas, 2008, p. 30) est très différent du sous-titrage interlinguistique ou «classique» d'une langue étrangère qui concerne le passage d'une langue à une autre langue. Or, ce qui fait la spécificité du sous-titrage pour malentendants c'est qu'il s'agit d'une traduction intralinguistique. En effet, comme l'indique Díaz Cintas (2008, p. 30):

Le contenu oral du dialogue des acteurs est transformé en un discours écrit proposé sous forme de sous-titres d'un maximum de trois à quelquefois quatre lignes. [...] Parallèlement aux dialogues, ils comportent toutes les informations paralinguistiques qui contribuent au développement de l'histoire ou à la création d'une atmosphère à laquelle la personne sourde n'a pas accès par la bande son.

Selon Neves (2008, p. 46), le sous-titrage SM se définit comme «Tout type de soustitre qui en plus de représenter la parole, décrit également les effets sonores, la musique et identifie clairement celui qui parle (la source sonore)».

Cependant, Neves (2008, p.45) n'est pas tout à fait d'accord avec l'utilisation de l'expression «pour sourds et malentendants» car elle précise qu'il s'agit d'un groupe de personnes très hétérogène ayant «des compétences et des besoins très différenciés en tant que réceptrices du sous-titrage». En effet, cette forme de soustitrage concerne des personnes ayant des profils très divers:

Il faut être conscient que les sous-titres que nous fournissons, dans un format plus ou moins standardisé, se destinent en réalité à des personnes ayant des caractéristiques très hétérogènes, déterminées par le degré, la localisation, le type, la cause et le début de la surdité. [...] II est également impensable qu'on puisse produire un seul type de sous-titre, adéquat pour tous: pour les personnes nées sourdes et qui n'ont jamais entendu et pour celles qui ont acquis la surdité plus tard (avec des référentiels et une mémoire auditive); pour les personnes qui n'ont aucune audition et pour celles qui disposent d'un certain degré d'audition résiduelle; adéquat aussi pour les personnes qui, malgré la perte auditive, s'identifient à la 
communauté entendante, (et utilisent le langage oral pour communiquer), autant que pour celles qui appartiennent à la communauté sourde (dont la langue maternelle est la langue des signes).

Cette traductologue constate que le manque de délimitation du public cible reste un écueil pour le sous-titreur SM. II est pratiquement impossible de produire un unique sous-titrage SM valable pour un public si diversifié ayant un réel besoin d'accès aux produits audiovisuels.

\section{Publicité et sous-titrage SM}

D'un point de vue linguistique, la publicité et le sous-titrage SM ont un point commun: ils jouent avec les mots en permanence. II faut trouver les mots les plus synthétiques et ceux qui véhiculent le plus fort concept avec très peu de caractères. Le sous-titreur SM devra tenir compte non seulement des caractéristiques des signes verbaux, mais également des relations que ceux-ci entretiennent avec les signes non verbaux tels que l'image, la musique et les effets sonores, afin de garantir l'intelligibilité du contenu du message publicitaire (Pettit, 2008). Le soustitrage pour malentendants doit être une description du contenu sonore et ne doit en aucun cas «saturer» le spectateur malentendant (Arnaiz Urquiza, 2012). Cependant, certaines campagnes publicitaires sont incompréhensibles sans les sous-titres pour malentendants et peuvent créer des «mal(entendus)» parce que les messages véhiculés présentent un type de scénarisation et des dialogues qui sont incompréhensibles pour le spectateur sourd et malentendant (Cambra, Silvestre \& Leal, 2009). Par exemple, les spots qui reproduisent le discours oral en décrivant les avantages et les inconvénients d'un service, par exemple, Gaz Réseau Distribution France $(G r D F)^{10}$, ou qui misent l'axe du produit sur l'aspect sonore uniquement: par exemple, le spot pour la Peugeot 208 Gti met en scène une jeune femme dans la salle de bains qui pose son pied sur une balance tout en émettant le son VROOM. Cette scène présente une situation incompréhensible pour le malentendant car elle est axée sur la reproduction du bruit d'accélération du moteur. Afin de le rendre accessible pour le spectateur sourd, ce spot devrait miser sur un autre axe de communication, par exemple, la sécurité ou le confort de l'automobile ${ }^{11}$.

Voyons ci-après un autre exemple de message publicitaire difficile d'accès pour le public malentendant qui devrait être réadapté: la publicité pour les céréales Miel Pops qui n'utilise que des onomatopées: «Miel Pops, c'est bzzz, splash, crunch,

\footnotetext{
10

<https://www.youtube.com/watch?v=Ez9CZ4TcCWY>

11

<https://www.youtube.com/watch?v=LQ50KnOfSys>
} 
miam». Ces onomatopées devraient être remplacées par des sous-titres qui soient pertinents pour les besoins sensoriels des malentendants, par exemple, les qualités gustatives du produit «bon miel d'abeilles» ${ }^{12}$.

Voici encore une publicité qui présente un obstacle à la compréhension pour les malentendants, le spot pour la Renault Mégane French Touch où la berline est présentée en empruntant des mots ou des expressions de langues différentes: «Das voiture», «Warum», «Splendido», «la touche francese», «Shocking», «So raffiné», «M. Van der Bruck», et en utilisant des jeux de mots «les belges font beaucoup d'autodérision». Dans ce spot, l'acteur imite tour à tour l'accent allemand, italien, anglais et belge sur un ton moqueur ce qui échappe évidemment au public malentendant ${ }^{13}$.

Ces quelques exemples démontrent le besoin urgent pour les annonceurs de réadapter leurs messages publicitaires au monde actuel où la tendance est à une communication de plus en plus visuelle, voire sensorielle, ainsi qu'au public malentendant. Selon De Linde \& Kay (1999), parmi les stratégies pour reproduire les sons dans les sous-titres SM se trouvent les suivantes: les étiquettes avec le nom des personnages, l'utilisation d'icônes, l'utilisation de couleurs pour identifier les parlants. Or, les créatifs publicitaires ont découvert de nombreuses alternatives visuelles et sensorielles et, en ce qui concerne le sous-titrage SM, la recherche académique se dirige également vers cette voie, notamment, les travaux de Civera \& Orero (2010) et de Tamayo (2015).

Il est possible d'associer le produit à des codes chromatiques comme dans le cas de la publicité pour le vernis à ongles OPI qui crée un nouvel alphabet avec des couleurs dont la force se trouve dans le slogan «Color is the universal language» (la couleur est le langage universel) $)^{14}$.

De nombreuses marques internationales déploient leurs services grâce aux nouvelles formes d'interaction comme Twitter. II est, par exemple, courant de nos jours de commmander avec un simple click sur le téléphone mobile grâce aux applications lancées sur Twitter, une pizza de Domino's Pizza, des frites de Burguer King, des hamburguers de Mc Donald's ou des chaussures de sport de Foot Locker ${ }^{15}$.

\footnotetext{
12 <https://www.youtube.com/watch?v=eNU9AZZEnig>

13 <https://www.youtube.com/watch?v=wA6p3mobKUQ>

14 <http://www.danstapub.com/une-marque-de-vernie-cree-un-nouvel-alphabet-avec-descouleurs/>

15 Voir l'article: <http://www.danstapub.com/pourquoi-les-emoticones-sont- ils-tendances-en2015/>
} 
La chaîne de restaurants Tim Hortons a créé des émoticônes symbolisant le Canada: les pictogrammes représentent un élan, un castor, une feuille d'érable, et des expressions typiques. Cette campagne apparait sur Twitter avec le hashtag \#TextLikeaCanadian. On peut également soutenir une bonne cause grâce à Lifeball contre le sida, ou dénoncer la maltraitance infantile avec l'association suédoise Bris ou faire des dons à $W W F$, la célèbre association pour la protection des espèces en voie de disparition qui a lancé une campagne de sensibilisation sur Twitter, avec le hashtag \#Endangeredemojis.

Mais, l'un des piliers de la communication actuelle, à la fois signe et symbole, et qui est très synthétique puisqu'il concentre en lui toutes sortes d'émotions, est l'émoticône. En 2015, elles ont créé tendance et sont devenues «le nouveau vocabulaire interactif des marques» ${ }^{16}$. Conscients de la vague d'émoticônes déversés quotidiennement sur WhatsApp, Facebook Messenger, Viber, WeChat, Line, les grands annonceurs n'ont pas d'autre solution, s'ils veulent atteindre les consommateurs, que d'utiliser ce nouveau champ de communication digitale.

En effet, les grandes marques telles que Coca-Cola, Pepsi, Dove, Starbucks ou Mc Donald's utilisent les émoticônes dans leurs campagnes publicitaires comme, par example, le spot «Venez comme vous êtes» de cette célèbre chaîne de restauration rapide $^{17}$. D'autres announceurs déploient leur propre application d'émoticônes à télécharger gratuitement sur les téléphones mobiles. C'est aussi le cas de la marque Ikea qui a lancé une application gratuite avec des émoticônes pour communiquer dans le couple. Le géant suédois crée ainsi un vocabulaire propre à la marque ${ }^{18}$. La marque d'automobiles Chevrolet a diffusé un communiqué de presse exclusivement à l'aide d'émoticônes. Et la marque Taco Bell a même fait une pétition pour que le «taco» devienne une émoticône universelle! L'incontournable marque Durex a lancé une campagne ${ }^{19}$ pour inclure un émoticône symbolisant un préservatif sur WhatsApp avec le hashtag \#emojicondom.

L'innovation atteint des sommets de créativité puisque certaines marques vont jusqu'à créer un langage propre afin de communiquer avec les consommateurs. Ceci est le cas de la marque Mentos qui a créé les «ementicons»: ce sont des émoticônes avec de nouvelles émotions quelque peu «décalées». La marque revendique la

\footnotetext{
16 À ce propos, voir l'article le Hub. Le média de la performance client (<http://www.laposte.fr/lehub/Les-emoticones-nouveau-vocabulaire>)

17 <https://www.youtube.com/watch?v=TY0pu35DENw>

18 <https://www.youtube.com/watch?v=UX02Ecfhndk>

19 <https://www.youtube.com/watch?v=07iKgKpkWfU>
} 
liberté d'expression avec le slogan suivant: «A way to express yourself» (Une façon de vous exprimer) ${ }^{20}$.

Tous ces exemples sont la preuve, qu'en publicité, s'adapter aux nouvelles technologies est de rigueur d'autant plus que cela créé une connivence entre la marque et le public cible ce qui rend le message plus efficace ${ }^{21}$. En ce qui concerne notre objet d'étude, le sous-titrage SM, l'utilisation des émoticônes peut être d'une grande utilité afin d'intégrer la communauté sourde et malentendante dans le flot des consommateurs digitalisés et potentiels.

\section{Optimiser le sous-titrage SM: les émoticônes animées}

Actuellement, l'émotion est au coeur des campagnes publicitaires et les émoticônes sont une source de plus en plus exploitée par les publicistes. De nombreux annonceurs qui utilisent des émoticônes dans leurs campagnes publicitaires le font à travers des applications mobiles dans le but de toucher les consommateurs d'aujourd'hui munis d'un smartphone car ce langage synthétique fait partie intégrante de notre vie quotidienne. Voyons un autre exemple de cette démarche qui devrait être la tendance à suivre par tous les annonceurs, mais, cette fois, sur un autre support médiatique très populaire: la télévision. II s'agit des campagnes du groupe Ferrero.

\subsection{Le cas de Ferrero}

Ferrero est une entreprise agroalimentaire italienne (piémontaise) solidement ancrée aux valeurs familiales et cela se ressent dans l'image véhiculée. C'est une entreprise familiale dont les fondateurs décidèrent d'élaborer des produits destinés à partager des moments de convivialité en famille. La stratégie de communication du groupe Ferrero consiste ainsi à conserver comme valeurs distinctives la tradition et l'esprit familial. Les marques les plus connues du groupe sont: Nutella, Ferrero Rocher, Mon Chéri, TicTac, Kinder. Concrètement, la marque Kinder couvre tous les besoins de la famille, Kinder Surprise, Kinder Chocolat, Kinder Délice, Kinder Pingui, Kinder Bueno, Kinder Country, Kinder Maxi, etc. et tous les produits sont présents dans 53 pays. En ce qui concerne le produit Kinder Chocolat, Yuste Frias (2011b, p. 271-77) a mis en évidence que les différentes stratégies paratraductives liées à l'image de

$<$ https://www.youtube.com/watch?v=VWVKshNYSwo>

21 Pour un aperçu sur les émotions en publicité, voir l'étude <http://www.danstapub.com/limpactdes-emotions-dans-la-communication/> 
l'enfant sur le packaging sont utilisées pour atteindre un seul et même but: l'achat. L'imaginaire du produit Kinder est construit autour des bienfaits nutritifs du lait (plus de lait, moins de cacao), ce qui est très approprié pour les enfants en pleine croissance, ceux-ci en étant les principaux consommateurs. En 2011, le groupe Ferrero, conscient du potentiel que constitue la cible des enfants sourds -ou plutôt leurs mères- décide de confier ses campagnes à l'agence IMD afin de permettre aux familles sourdes françaises d'accéder à tous ses produits. À partir de cette date cruciale, tous les spots de Ferrero sont sous-titrés pour sourds et malentendants par l'agence IMD dont les principales contraintes du processus de création-livraison d'une campagne ${ }^{22}$ sont les suivantes:

«Minimiser l'occupation de l'espace visuel

- Rappel: les sous-titres ne sont visibles que si activés

- Respect de la création originale

Positionnement des sous-titres

- Jamais devant les mentions légales

- Jamais devant logo ou éléments graphiques essentiels

Un équilibre délicat et nécessitant une grande expertise

- Respect de la charte vs montage rapide vs nombreux évènements audio vs occupation de l'image

- Respect du langage de la marque

- Personnels spécialisés sous-titrage SM + formation publicité TV»

À présent, nous allons nous attarder sur un spot de la campagne publicitaire télévisée du groupe Ferrero, à savoir, le spot pour la barre chocolatée Kinder Bueno.

\subsection{Analyse du spot Kinder Bueno}

À titre représentatif, nous allons analyser le spot pour le produit Kinder Bueno en français ${ }^{23}$ ainsi que la version française sous-titrée pour malentendants. Le soustitrage SM du spot pour le produit Kinder Bueno a été aimablement fourni par la société IMD Media Limited France à Paris. IMD est une société de services qui est à l'origine de la livraison numérique de films publicitaires télévisés en France et elle assure la livraison des films vers les principales régies publicitaires et les chaînes télévisées dont elles commercialisent l'espace. Parmi les clients les plus importants en volume de travail se trouvent Ferrero, l'Oréal et GMF, pionniers dans le soustitrage SM en France depuis 2011.

\footnotetext{
22 Consignes explicites de l'équipe de IMD Paris (Sous-titrage SM-Audition CPP. 24/10/2014).
}

23 <https://www.youtube.com/watch?v=0hZw10zByow> 
Dans le tableau 1 ci-après, nous restituons les dialogues pour la version française (VF) ainsi que la version française sous-titrée pour sourds et malentendants (VFSM) du spot pour le produit Kinder Chocolat.

\begin{tabular}{ll}
\hline VF (Version française) & $\begin{array}{l}\text { VFSM (Version française sous-titrée } \\
\text { pour sourds et malentendants) }\end{array}$ \\
\hline -Oh, un Bueno! & Un Bueno? \\
\hline -Oui, c'est le dernier! & -Le dernier! \\
\hline Dommage pour toi! & Dommage, hein? \\
\hline Ah, ben c'est cool. Tu l'as bien pris! & Ah, tu l'as bien pris! C'est cool. \\
\hline Ben quoi? & Quoi? \\
\hline Ben ta nouvelle voiture abîmée par & Ta voiture abîmée par maman! \\
maman! & \\
\hline & Il souffle \\
\hline Un coeur fondant aux noisettes, & Un coeur fondant aux noisettes, \\
\hline Une gaufrette croustillante. & Une gaufrette croustillante. \\
\hline Du bon chocolat. & Du bon chocolat. \\
\hline Kinder Bueno & Kinder Bueno... \\
\hline Si bon qu'on en deviendrait méchant. & \\
\hline
\end{tabular}

Tableau 1. Transcription des dialogues en français et des sous-titres français pour sourds et malentendants du spot TV pour le produit Kinder Bueno.

Dans la version sous-titrée SM, nous pouvons observer que les normes en vigueur ont été scrupuleusement respectées: d'un point de vue linguistique, le sous-titreur SM a eu recours à des structures syntaxiques simples: «Ah, ben c'est cool. Tu l'as bien pris!» / «Ah, tu l'as bien pris! C'est cool.», à une sélection du vocabulaire («croustillante» / «craquante») dû aux exigeances de l'annonceur de maintenir l'adjectif «croustillante» malgré le nombre de caractères (12/8); à des exclamations plus synthétiques: «Oui, c'est le dernier!» / «Le dernier!»; «Dommage pour toi!» / «Dommage!»; ainsi qu'à des constructions où les marques d'oralité disparaissent «Ben quoi?» / «Quoi?». En ce qui concerne l'indentification des parlants, celle-ci est facilitée grâce au positionnement des sous-titres qui sont placés sur la partie inférieure de l'écran à gauche ou à droite selon la personne qui parle. Les informations complémentaires sur les états d'âme des personnages telles que le soupir du père sont restituées en rouge: «ll souffle». Le sous-titreur SM utilise ces conventions dans le but de permettre au spectateur sourd un moindre effort de décodification et une lecture plus rapide et aisée. En effet, selon Neves (2008: p. 50): «Une des plus grandes préoccupations des sous-titreurs est de créér des sous-titres faciles à lire, sachant que les personnes atteintes de surdité ont une difficulté plus grande de lecture». 
Les propos des personnages, la musique, les effets sonores et l'identification des parlants, bref, toutes les conventions sont respectées dans ce sous-titrage SM. Cependant, même si toutes ces informations sont correctement restituées, le spot demeure encore axé sur la lecture des sous-titres. Fort heureusement, à la fin du spot, l'apparition d'une émoticône animée permet d'attirer rapidement l'attention du spectateur malentendant grâce à un clin d'œil «animé» (voir Figure 1, ci-dessous):

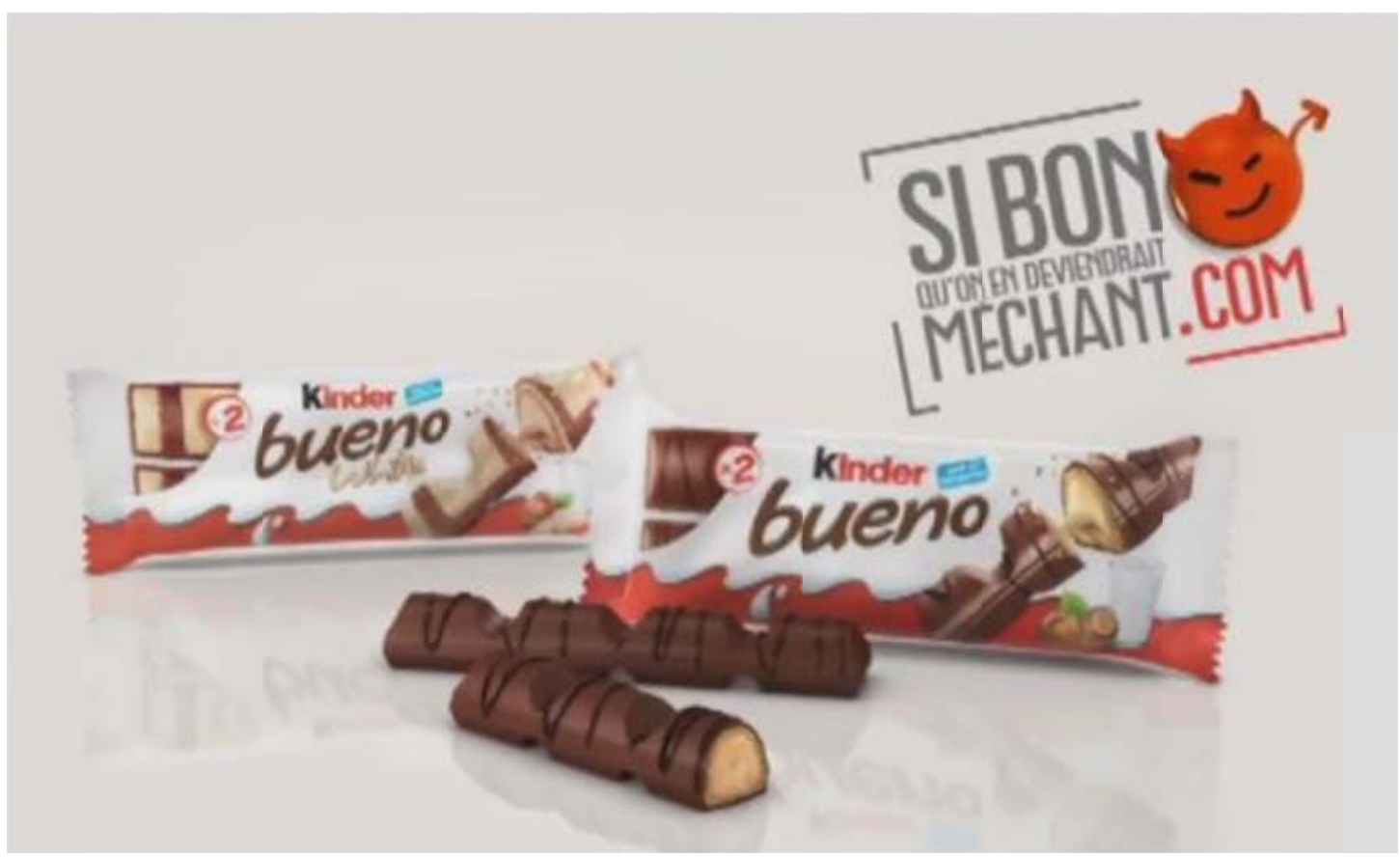

Figure 1. Version française du spot pour le produit Kinder Bueno.

Les émoticônes sont ce que Yuste Frias $\left(2016^{24}\right)$ nomme des «soupirs du texte» car elles proviennent, en fait, des signes de ponctuation. Elles ont été créées pour «para-traduire» des émotions et des états d'âme à travers la messagerie instantanée. Dans notre exemple, l'émoticône animée du visage souriant provient des signes de ponctuation suivants: deux points, tiret et parenthèse. Ici, l'émoticône animée du visage souriant se transforme en diable pour ainsi assurer la cohérence du spot au niveau verbal et non-verbal (Cruz García, 2004; Valdés Rodríguez \& Fuentes Luque, 2008). La force persuasive de l'émoticône animée se situe dans le passage du sourire "gentil» (Kinder Bueno = bon) au sourire «malim» (Kinder Bueno rend méchant) mis en valeur par l'antithèse du slogan «Si bon qu'on en deviendrait méchant». Jeux de mots, jeux d'images... Grâce à ce clin d'œil humoristique, le spot contribue à une excellente compréhension du message en dépit du degré ou du 
type de surdité (Neves, 2008). En outre, la combinaison du slogan et de l'image ne visent pas seulement la compréhension du message, ils constituent également «un moyen efficace de mémorisation» (Guidère, 2000, p. 240).

L'exemple de la publicité Kinder Bueno du groupe Ferrero que nous avons analysé est représentatif de la tendance actuelle des annonceurs publicitaires à sur-solliciter l'aspect visuel. Cette tendance bénéficie autant le public entendant que le public malentendant puisqu'elle implique, d'une part, un rapport spécifique à l'image et, d'autre part, elle attire l'attention du spectateur malentendant en combinant la lecture d'un texte bref au visionnage d'une image animée. D'après Gottlieb (1994), le succès du sous-titrage dépend du lien créé par rapport à l'image tout comme la relation des dialogues avec l'image dans la version originale.

Or, il convient d'intégrer le public des malentendants dans la masse de consommateurs potentiels, ils ont eux aussi le «droit» d'être informés par les médias de la même façon que le public entendant. Si les publicistes souhaitent intégrer le public sourd et malentendant en tant que consommateur potentiel, ils doivent réadapter leurs campagnes aux goûts du jour car, comme le rappelle Dávila Montes (2008), le but de la publicité est de persuader les acheteurs potentiels d'acquérir et de consommer le produit.

\section{Conclusion}

Sans avoir pu traiter de manière exhaustive tous les aspects du sous-titrage SM, ce travail a démontré la nécessité fondamentale d'adapter les films publicitaires non seulement à la vie actuelle qui est de moins en moins versée dans la lecture mais plus dans le visuel, mais surtout de les réadapter à un public cible particulier: les sourds et malentendants. Dialogues interminables, onomatopées inconnues, accents étrangers, structures syntaxiques complexes, métaphores, jeux de mots, autant de messages publicitaires incompréhensibles qui supposent un vrai «calvaire» pour les sourds et malentendants (Herrera et al., 2007). Le principal défi pour les annonceurs est de réadapter constamment la stratégie de communication de la marque aux temps et aux mœurs, de miser sur des messages publicitaires qui font de plus en plus appel à des associations visuelles, sensorielles, voire «synesthésiques» et d'investir dans l'accessibilité afin que les malentendants puissent «écouter les sous-titres» (Matamala \& Orero, 2010). Pour ce qui est de la labeur du sous-titreur professionnel, il s'agit pour lui de trouver un délicat équilibre necessitant une grande expertise, un compromis stratégique entre le langage de la marque et le public malentendant dont dépendra le succès ou l'échec de la campagne publicitaire. La marge de manœuvre du sous-titreur SM est réduite mais s'il adapte les sous-titres avec créativité aux besoins spécifiques et réels du public 
malentendant, il y a de fortes chances pour que le résultat se traduise dans la compréhension globale du message et, par conséquent, soit à l'origine de la nécessaire inclusion des malentendants en tant que consommateurs à part entière.

Au terme de ce travail consacré au sous-titrage des films publicitaires pour sourds et malentendants, nous espérons avoir contribué à la sensibilisation de cette modalité de traduction audiovisuelle qui connait un intérêt académique croissant (Díaz Cintas et al., 2010; Romero Fresco, 2015; Tamayo, 2015) ouvrant ainsi la voie pour de futures recherches et des perspectives d'avenir professionnel assez encourageantes.

\section{About the author}

Isabel Cómitre Narváez holds a Master's degree in Applied Linguistics and Trade (English/Spanish) from the University of Toulouse (France) and a Ph.D. in Translation Studies from University of Málaga, where she has taught translation theory and practice since 1994 in the Department of Translation and Interpretation. She has published articles on translation and foreign languages, translation of advertising and audiovisual translation.

\section{Acknowledgements}

Ce travail s'inscrit dans le cadre du projet de recherche Erasmus+:"Enhancing communication: research to improve communication for people with special needs and development of ICT resources and tools" (2015-1-ES01-KA203-015625) sous la direction de la docteure Encarnación Postigo Pinazo <https://sites.google.com/site/ecplusproject/>.

Je tiens à remercier toute l'équipe parisienne pour son chaleureux accueil et son inestimable collaboration: M. Jean Gaillard, représentant en France d'IMD Media Limited; M. Julien Soret, directeur des opérations; M. Nicolas Letier-Lacaze, directeur commercial de la clientèle; Mlle. Marcela Rodriguez, Melle. Marie Terrier et M.Jean-Louis Buchet, chargés d'affaires. 


\section{LFE Article history}

Paper received: 1st September 2015

Paper received in revised form and accepted for publication: 22th April 2016

\section{Références bibliographiques}

Arnaiz Urquiza, V. (2012). Los parámetros que identifican el subtitulado para sordos. Análisis y clasificación. MonTI, 4, 103-132.

Asociación Española de Normalización y Certificación (AENOR) (2012). Norma UNE 153010 Subtitulado para personas sordas y personas con discapacidad auditiva. Madrid: AENOR.

British Broadcasting Corporation (BBC) (2009). Online Subtitling Editorial Guidelines. <http://www.bbc.co.uk/guidelines/futuremedia/accessibility/subtitling_guides/online_sub _editorial_guidelines_vs1_1.pdf> [15/08/2015].

Conseil Supérieur de l'Audiovisuel (CSA) (2011). Charte relative à la qualité du sous-titrage à destination des personnes sourdes ou malentendantes. <http://www.csa.fr/en/Espacejuridique/Chartes/Charte-relative-a-la-qualite-du-sous-titrage-a-destination-despersonnes-sourdes-ou-malentendantes-Decembre-2011> [20/08/2015].

Cambra, C., Silvestre, N. \& Leal, A. (2009). Análisis de la comprensión por parte del alumnado sordo de los documentos televisivos subtitulados y criterios de mejora. Quaderns del CAC, 31-32, 155-159.

Civera, C. \& Orero, P. (2010). Introducing icons in subtitles for the deaf and hard of hearing: Optimising reception. In A. Matamala \& P. Orero (Eds.), Listening to subtitles. Subtitles for the deaf and hard of hearing (pp. 149-162). Bern, Berlin, Bruxelles, Frankfurt am Main, New York, Oxford, Wien: Peter Lang.

Cruz García, L. (2004). Características diferenciales de la traducción publicitaria. El papel del traductor de anuncios. In L. Lorenzo \& A. Pereira (Eds.), Traducción subordinada III: Traducción y publicidad (pp.17-28). Vigo: Servicio de Publicaciones de la Universidad de Vigo.

Dávila Montes, J. (2008). La traducción de la persuasión publicitaria. Lewiston/Queenston/Lampeter: The Edwin Mellen Press.

De Linde, Z. (1996). Le sous titrage intralinguistique pour les sourds et les malentendants. In Y. Gambier (Ed.), Les transferts linguistiques dans les médias audiovisuals (pp. 165-83). Paris: Presses Universitaires du Septentrion.

De Linde, Z. \& Kay, N. (1999). The semiotics of subtitling. Manchester: St. Jerome.

Díaz Cintas, J. (2008). Pour une classification des sous-titres à l'époque du numérique. In J.M. Lavaur \& A. Serban (Eds.), La traduction audiovisuelle. Approche interdisciplinaire du sous- 
titrage (pp. 27-41). Bruxelles: De Boeck.

Díaz Cintas, J., Matamala Ripoll, A. \& Neves, J. (2010) (Eds.) New insights into audiovisual translation and media accessibility. Amsterdam: Rodopi.

Gottlieb, H. (1994). Translating people, not words. Subtitling - visual interpretation. In C. Dollerup \& A. Lindegaard (Eds.), Teaching Translation and Interpreting. Insights, aims, visions (pp. 261-274). Amsterdam: John Benjamins.

Guidère, M. (2000). Publicité et traduction. Paris: L'Harmattan.

Herrera, V., Puente, A., Alvarado, J. M. \& Ardilla, A. (2007). Códigos de lectura en sordos: la dactilología y otras estrategias visuales y kinestésicas. Revista latinoamericana de Psicología, 39(2), 269-286.

Neves, J. (2008). Le sous-titrage pour sourds et malentendants: à la recherche d'une qualité possible. In J.M. Lavaur \& A. Serban (Eds.), La traduction audiovisuelle. Approche interdisciplinaire du sous-titrage (pp. 43-54). Bruxelles: De Boeck.

Pereira, A. (2010). Criteria for elaborating subtitles for deaf and hard of hearing adults in Spain: Description of a case study. In A. Matamala \& P. Orero (Eds.), Listening to subtitles. Subtitling for the Deaf and Hard of Hearing (pp. 87-102). Frankfurt, Berlin, Bern, Bruxelles, New York, Oxford, Wien: Peter Lang.

Pereira, A. \& Lorenzo, L. (2005). Evaluamos la norma UNE 153010: subtitulado para personas sordas y personas con discapacidad auditiva. Subtitulado a través del teletexto. Puentes, 6, 21-26.

Pettit, Z. (2008). Le sous-titrage: le rôle de l'image dans la traduction d'un texte multimodal. In J.M. Lavaur \& A. Serban (Eds.), La traduction audiovisuelle. Approche interdisciplinaire du sous-titrage (pp. 101-11). Bruxelles: De Boeck.

Romero Fresco, P. (2015) (Ed.) The Reception of Subtitles for the Deaf and Hard of Hearing in Europe, UK, Spain, Italy, Poland, Denmark, France and Germany. Frankfurt, Berlin, Bern, Bruxelles, New York, Oxford, Wien: Peter Lang.

Tamayo, A. (2015). Estudio descriptivo y experimental de la subtitulación en TV para niños sordos. Una propuesta alternativa. Tesis doctoral (inédita). Castellón de la Plana: Universitat Jaume I.

Valdés Rodríguez, C. (2004). La traducción publicitaria, comunicación y cultura. Valencia: Universidad de Valencia.

Valdés Rodriguez, C. \& Fuentes Luque, A. (2008). Coherence in translated television commercials. European Journal of English Studies, 12, 133-148.

Yuste Frías, J. (2010). Au seuil de la traduction: la pararaduction. In T. Naaijkens (Ed.), Event or Incident. Événement ou Incident. On the Role of Translation in the Dynamics of Cultural Exchange. Du rôle des traductions dans les processus d'échanges culturels, 3 (pp. 287-316). Bern, Berlin, Bruxelles, Frankfurt am Main, New York, Oxford, Wien: Peter Lang, col. Genèses de Textes-Textgenesen (F. Lartillot [dir.]).

Yuste Frías, J. (2011a). Traducir para la pantalla: el traductor entre el texto y la imagen. En E. 
Di Giovanni (Ed.), Diálogos intertextuales 5: Between Text and Receiver: Translation and Accessibility. Entre texto y receptor: traducción y accesibilidad (pp. 57-88). Frankfurt, Berlin, Bern, Bruxelles, New York, Oxford, Wien: Peter Lang.

Yuste Frias, J. (2011b). Leer e interpretar la imagen para traducir. Trabalhos em Lingüística Aplicada (TLA), 50(2), 257-80. <http://www.scielo.br/pdf/tla/v50n2/03.pdf> [28/08/2015].

Yuste Frías, J. (2013). Nuevas perspectivas para traducir la imagen en publicidad internacional. In X. Montero Dómínguez (Ed.), Traducción para la comunicación internacional (pp. 83-92). Granada: Comares.

Yuste Frías, J. (2015). Paratraducción: la traducción de los márgenes, al margen de la traducción. Delta, 31, 317-347. 